Direct Numerical Simulation of Chemical Non-equilibrium Turbulent Flow

This content has been downloaded from IOPscience. Please scroll down to see the full text. 2013 Chinese Phys. Lett. 30064702

(http://iopscience.iop.org/0256-307X/30/6/064702)

View the table of contents for this issue, or go to the journal homepage for more

Download details:

IP Address: 159.226.199.81

This content was downloaded on 21/09/2013 at 12:33

Please note that terms and conditions apply. 


\title{
Direct Numerical Simulation of Chemical Non-equilibrium Turbulent Flow *
}

\author{
CHEN Xiao-Ping(陈小平) $)^{1,2}$, LI Xin-Liang(李新亮 $)^{1 * *}$ \\ ${ }^{1}$ Key Laboratory of High Temperature Gas Dynamics, Institute of Mechanics, Beijing 100190 \\ ${ }^{2}$ School of Mechanical Engineering and Automation, Zhejiang Sci-Tech University, Zhejiang 310018
}

(Received 26 December 2012)

\begin{abstract}
Temporally evolving high-temperature turbulent channel flows (at $M a_{\infty}=6$ and 10 and $R e_{\infty}=12000$ ) are performed by using direct numerical simulation with the assumption of local thermal equilibrium and chemical non-equilibrium. The turbulent statistical characteristics are studied. We find that the Morkovin theory for the Van Direst transformed velocity remains valid, while the compressibility effects need to be considered since the turbulent Mach number is high enough, especially for the higher Mach number case. The dissociation/recombination reactions are excited, which are proved by the mean temperature, mass fractions and specific heat ratio. The importance of the mean property variations is studied from the rms velocity and mass fraction fluctuations.
\end{abstract}

PACS: 47.27.E- 34.50.Lf, 47.40.Ki, 47.70.Nd

Turbulent flow induces a much higher thermal load on the surface of vehicles re-entering a planetary atmosphere on comparison to laminar. ${ }^{[1]}$ In hypersonic flight, shock waves and large kinetic dissipation make the temperature extremely high, which leads to realgas effects. The vehicle surface will be covered by chemical reactions. Therefore, in order to predict the hypersonic turbulent characteristics accurately, it is necessary to consider the non-equilibrium effects. ${ }^{[2]}$

Study of turbulent characteristics with a focus on non-equilibrium flows have mostly been based on experiments or linear theories for parallel and nonparallel flow. In a turbulent flow simulation, compared with large-eddy simulation (LES) and Reynolds average Navier-Stokes (RANS), direct numerical simulation (DNS) solves the Navier-Stokes equations directly and does not contain any modeling errors, and is a powerful tool to study the mechanism of turbulent flows. Many direct numerical simulations for finite-rate chemistry have been reported for flows involving combustion. However, those simulations are mainly focused on the low Mach number flows, which have some difference from this study. The reports of DNS for turbulent flows with chemical non-equilibrium in high Mach number are relatively few. Martin et al. ${ }^{[3-8]}$ use simplified single dissociation/recombination reaction mechanisms and constant species heat capacities to study the nonlinear interaction between turbulence and finite-rate chemical reactions in the presence of predominantly exothermic or endothermic reactions in isotropic turbulence and turbulent boundary layers. Duan et al. ${ }^{[9]}$ study and assess the presence and effects of turbulence-chemistry interaction for hypersonic conditions in the Earths atmosphere. Duan et al. ${ }^{[10]}$ perform DNS of flat-plate hypersonic turbulent boundary layers at high- and

\section{DOI: $10.1088 / 0256-307 \mathrm{X} / 30 / 6 / 064702$}

low-enthalpy conditions on air to study the effects of enthalpy conditions on boundary layer flow. Simultaneously, Marxen et al. ${ }^{[11,12]}$ have carried out some research, for example, using different gas-modes to analyze the problem of hypersonic boundary layer stability.

All these studies are about isotropic or boundary layers turbulence, and do not involve the internal flows. However, in Scramjet, turbulent flows with nonequilibrium effects are also very important. Based on the engineering background, direct numerical simulations for a three-dimensional channel with chemical non-equilibrium effects are performed.

The code is developed from our foregoing works, ${ }^{[13]}$ assumed to be thermally perfect gas and chemically reacting gas in equilibrium. The governing equations are the time-dependent three-dimensional Navier-Stokes equations ${ }^{[14]}$ for a compressible fluid in non-dimensional form. These equations are formulated for a mixture of thermally perfect gases in local thermal equilibrium, where chemical reactions are assumed to take place with a finite reaction rate, while vibrational relaxation occurs infinitely fast.

$$
\begin{aligned}
& \frac{\partial \rho}{\partial t}+\frac{\partial}{\partial x_{j}}\left(\rho u_{j}\right)=0, \\
& \frac{\partial \rho_{s}}{\partial t}+\frac{\partial}{\partial x_{j}}\left(\rho_{s} u_{j}-\rho_{s} D_{s} \frac{\partial Y_{s}}{\partial x_{j}}\right)=w_{s}, \quad s=1,2, \cdots n s-1, \\
& \frac{\partial\left(\rho u_{i}\right)}{\partial t}+\frac{\partial}{\partial x_{j}}\left(\rho u_{i} u_{j}+p \delta_{i j}-\sigma_{i j}\right)=0, i=1,2,3, \\
& \frac{\partial E}{\partial t}+\frac{\partial}{\partial x_{j}}\left((E+p) u_{j}-u_{i} \sigma_{i j}+q_{j}-\sum_{s} \rho_{s} D_{s} \frac{\partial Y_{s}}{\partial x_{j}} h_{s}\right)=0,
\end{aligned}
$$

where $\rho_{s}$ is the density of species $s, \rho$ is the total mix-

*Supported by the National Natural Science Foundation of China under Grant No 11072248, the National Basic Research Program of China under Grant No 2009CB724100, the National High-tech Research and Development Program of China under Grant No 2012AA01A304, and the CAS Program under Grant No KJCX2-EW-J01.

${ }^{* *}$ Corresponding author. Email: lixl@imech.ac.cn

(C) 2013 Chinese Physical Society and IOP Publishing Ltd 
ture velocity, $Y_{s}=\rho_{s} / \rho$ is the mass fraction of species $s, u_{j}$ is the mass-averaged velocity in the $j$ direction, $\sigma_{i j}$ is the shear stress tensor, and $p$ is the pressure, which is given by

$$
p=\sum_{s} \rho_{s} \frac{R}{M_{s}} T
$$

where $R$ is the universal gas constant $\left(8.314 \mathrm{~J} \cdot \mathrm{mol}^{-1} \mathrm{~K}^{-1}\right), h_{s}$ is the enthalpy of species $s$, and $q_{j}$ is the total conductive heat flux which is described by Fourier's law. The total energy $E$ is given by

$$
E=\sum_{s=1}^{n s} \rho_{s} e_{s}+\frac{1}{2} \rho u_{i} u_{i}
$$

where $e_{s}$ is the species internal energy per unit mass, which includes the translational, rotational and vibrational energy and can be seen in Ref. [1], ignoring the electronic energy.

Due to the fact that theoretical solutions of the transport properties are extremely complex, semiempirical formulas and curve fits will be used. The species viscosity $\mu_{s}$ is taken from the work of Gupta and obtained from the following curve fit. ${ }^{[15]}$ The thermal conductivity of species $s$ is computed using Eucken's semi-empirical formula. ${ }^{[16]}$ A common mixture rule for viscosity and thermal conductivity is Wilke's rule. ${ }^{[15,16]}$ The Schmidt number $S c$ is assumed to be the same constant for all the species, whose value is 0.5. The kinematic diffusion coefficient $D_{s}$ is then computed from the definition:

$$
D_{s}=\frac{1}{\rho} \frac{\left(1-Y_{s}\right) \mu}{\left(1-X_{s}\right) S c} .
$$

In this study, the chemistry model is that of air consisting of five species $(n s=5)$. This model represents realistic reactions of air in the absence of ionization and is a good approximation at temperatures less than about $10000 \mathrm{~K}$. The constituent species are molecular oxygen $\left(\mathrm{O}_{2}\right)$, atomic oxygen $(\mathrm{O})$, nitric oxide (NO), nitrogen $(\mathrm{N})$, and molecular nitrogen $\left(\mathrm{N}_{2}\right)$. The following reactions are considered, ${ }^{[17]}$

$$
\begin{aligned}
& \mathrm{O}_{2}+\mathrm{M} \longleftrightarrow 2 \mathrm{O}+\mathrm{M}, \mathrm{NO}+\mathrm{M} \longleftrightarrow \mathrm{N}+\mathrm{O}+\mathrm{M} \\
& \mathrm{N}_{2}+\mathrm{M} \longleftrightarrow 2 \mathrm{~N}+\mathrm{M}, \mathrm{N}_{2}+\mathrm{O} \longleftrightarrow \mathrm{NO}+\mathrm{N}, \\
& \mathrm{NO}+\mathrm{O} \longleftrightarrow \mathrm{O}_{2}+\mathrm{N}
\end{aligned}
$$

where $\mathrm{M}$ can be $\mathrm{O}_{2}, \mathrm{O}, \mathrm{NO}, \mathrm{N}$, and $\mathrm{N}_{2}$. The $k$ th reaction can be symbolically represented as

$$
\sum_{s=1}^{n s} \alpha_{k, s} A_{s} \longleftrightarrow \sum_{s=1}^{n s} \beta_{k, s} A_{s}
$$

where $\alpha_{k, s}$ and $\beta_{k, s}$ are the stoichiometric coefficients for reactants and products of species $s$, respectively, and $A_{s}$ is the chemical symbol of the $s$ th species. The mass productions/depletion rate of species $s, w_{s}$, can be defined to be

$w_{s}=M_{s} \sum_{k=1}^{n r}\left(\beta_{k, s}-\alpha_{k, s}\right)\left(k_{\mathrm{f}, k} \prod_{r=1}^{n s} c_{r}^{\alpha_{k, r}}-k_{\mathrm{b}, k} \prod_{r=1}^{n s} c_{r}^{\beta_{k, r}}\right)$,

where $c_{r}$ is the concentration of species $r, k_{\mathrm{f}, k}$ and $k_{\mathrm{b}, k}$ are the forward and backward reaction rate coefficients of the $k$ th reaction, respectively. The forward reaction rate coefficient can be determined from the Arrhenius expression. ${ }^{[18]}$ The backward reaction rate coefficient is given by

$$
k_{\mathrm{b}, k}=\frac{k_{\mathrm{f}, k}}{K_{\mathrm{eq}, k}},
$$

where the equilibrium constant $K_{\mathrm{eq}, k}$ is a function of $T$ and can be determined using curve fits. ${ }^{[19]}$
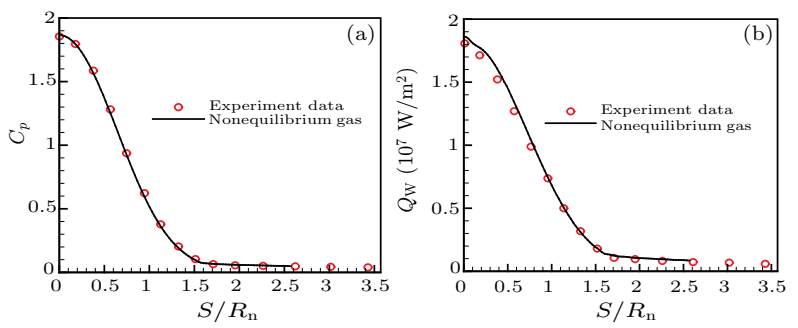

Fig. 1. (a) Surface pressure coefficient of cone. (b) Surface heating rates of cone.

In order to study the effects of chemical nonequilibrium on turbulence, direct numerical simulations are performed. The compressible Navier-Stokes equations are solved by using the Van-Leer flux vector splitting and the 7th-order $\mathrm{WENO}^{[20]}$ scheme to discrete convection terms. The viscous terms are approximated with an 8th-order central difference. A 3rd-order Runge-Kutta method is used for the time integration.

To test the code, we perform a test case for hypersonic non-equilibrium flow over a sharp cone. ${ }^{[21]}$ The geometry for the test case consists of zero degrees angle attack. Geometry and free stream conditions are as follows: ${ }^{[21]}$

$$
\begin{aligned}
R_{n} & =0.7 \mathrm{~cm}, U_{\infty}=3630 \mathrm{~m} / \mathrm{s} \\
T_{\infty} & =293 \mathrm{~K}, P_{\infty}=2400 \mathrm{~Pa}
\end{aligned}
$$

This is a typical verification example of the nonequilibrium code. The flow has a fully catalytic isothermal wall with $T_{\mathrm{w}}=1000 \mathrm{~K}$. Computation is a $91 \times 151$ grid (stream-wise direction $\times$ wall-normal direction), which is generated with algebraic method. The minimum grid spacing is $2.0 \times 10^{-4}$.

Figures 1(a) and 1(b) show surface pressure coefficients and surface heating rates, respectively. The calculated results produce a good agreement with experimental data.

The non-equilibrium model often corresponds to a particular physical problem, such as the flight height and velocity. In this study, the simulated flight height is $20 \mathrm{~km}$ and flight speed includes two situations. The 
non-dimensional parameters, related to the infinite free-stream flight conditions, are defined as

$$
R e_{\infty}=\frac{\rho_{\infty} U_{\infty} L}{\mu_{\infty}}, M a_{\infty}=\frac{U_{\infty}}{c_{\infty}},
$$

where $\rho_{\infty}, c_{\infty}, \mu_{\infty}$ represent the density, speed of sound, viscosity in the free-stream point, respectively, and $L$ is the half width of the channel. The specific value can be referenced to the earth standard atmosphere table, as shown in Table 1. Nondimensionalization is based on the free-stream conditions (marked by $\infty)$. Dimensionless characteristic time is $L / U_{\infty}$. It should be emphasized that the hypersonic inflow Mach number is not the real Mach number in channel flow, but the flight Mach number of hypersonic aircraft. The domain size $\left(L_{x} \times L_{y} \times L_{z}\right)$, the grid size $(x \times y \times z)$ and the number of grid points $\left(n_{x} \times n_{y} \times n_{z}\right)$ are given in Table 2, respectively. For wall turbulence, the streamwise length should be greater than 2000 wall scale, and spanwise length should be greater than 400 wall scale. ${ }^{[22]}$ The wall scale is defined as

$$
l^{+}=\frac{\mu_{\mathrm{w}}}{\rho_{\mathrm{w}} u_{\tau}} .
$$

The wall scale $\left(l^{+}\right)$is given by Table 4 . Compared with the experience, the computational domain is sufficient. The grid resolution $\left(x^{+} \times y^{+} \times z^{+}\right)$is similar to Duan et al. ${ }^{[10]}$ Boundary conditions and grids can be seen from Ref. [1]. The fully catalytic wall assumes infinitely fast atom recombination at the wall.

Table 1. Free-stream and wall parameters for the compressible turbulent channel flow.

\begin{tabular}{cc}
\hline Density $\rho_{\infty}\left(\mathrm{kg} / \mathrm{m}^{3}\right)$ & $8.891 \times 10^{-2}$ \\
Temperature $T_{\infty}(\mathrm{K})$ & 216.65 \\
Speed of sound $c_{\infty}(\mathrm{m} / \mathrm{s})$ & 295.07 \\
Wall temperature $T_{\mathrm{w} \infty}(\mathrm{K})$ & 1300 \\
Reynolds number $R e_{\infty}$ & 12000 \\
Mach number $M a_{\infty}$ & $6(\mathrm{M} 1), 10(\mathrm{M} 2)$ \\
\hline
\end{tabular}

Table 2. Grid resolution and domain size for the DNS data.

\begin{tabular}{cccccccccc}
\hline Cases & $L_{x}$ & $L_{y}$ & $L_{z}$ & $\Delta x^{+}$ & $\Delta y^{+}$ & $\Delta z^{+}$ & $n_{x}$ & $n_{y}$ & $n_{z}$ \\
\cline { 1 - 8 } M1 & \multirow{2}{*}{$4 \Pi$} & \multirow{2}{*}{2} & \multirow{2}{*}{$4 \Pi / 3$} & 22.4 & 0.43 & 7.47 & 161 & 201 & 161 \\
\cline { 5 - 9 } & & & & 24.2 & 0.45 & 8.01 & 201 & 261 & 201 \\
\hline
\end{tabular}

Chemical non-equilibrium source terms reflect the influence of chemical reactions, which will bring stiffness. In order to ensure the precision of time, an explicit time marching scheme has been used, which requires a very small time step. At the same time, due to the fact that the thermo-dynamic physical quantities and transport coefficients are not only the function of density and temperature, but also have relationship with components, the time in per step is very long. Time step and the time in per step are given in Table 3 . It can be seen that direct numerical simulations of such problems are very difficult. To save computing time and improve computational efficiency, the initial flow field is selected, a fully developed turbulent instantaneous field of Ref. [13] $\left(M a_{\infty}=6\right)$. Simulate 200 dimensionless times, and then begin the statistical analysis. Statistical time lasts about 50 dimensionless times. Figure 2 plots an instantaneous flow of atomic oxygen in a $(x, y)$ plane. In order to understand chemical non-equilibrium turbulent flow more intuitively, Fig. 3 shows a second invariant of the velocity gradient tensor.

Figure 4(a) depicts the relationship between the skin friction coefficients and the statistical time. The skin friction coefficient is defined as

$$
C_{f}=\frac{2 \tau_{\mathrm{w}}}{\rho_{\infty} u_{\infty}^{2}}, \quad \tau_{\mathrm{w}}=\left.\left\langle\mu_{\mathrm{w}}\right\rangle \frac{\partial\langle u\rangle}{\partial y}\right|_{\mathrm{w}} .
$$

Also included in Fig. 4(a) are the theoretical estimates for the fully turbulent regime given by White, ${ }^{[23]}$

$$
C_{f}=\frac{0.455}{S^{2}}\left[\log \left(\frac{0.06}{S} \operatorname{Rex} \frac{1}{\left\langle\mu_{\mathrm{w}}\right\rangle} \sqrt{\frac{1}{\left\langle T_{\mathrm{w}}\right\rangle}}\right)\right]^{-2},
$$

where

$$
\begin{aligned}
& S=\frac{1}{\sin ^{-1} A} \sqrt{\left\langle T_{\mathrm{w}}\right\rangle-1}, \\
& A=\left(r \frac{\gamma-1}{2} M a_{\infty}^{2} \frac{1}{\left\langle T_{\mathrm{w}}\right\rangle}\right)^{1 / 2} .
\end{aligned}
$$

The simulation shows a good agreement with the White correlation in the fully turbulent region.
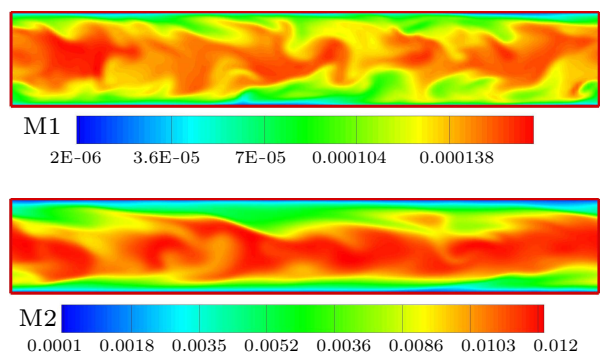

Fig. 2. Instantaneous flow of atomic oxygen in a $(x, y)$ plane.

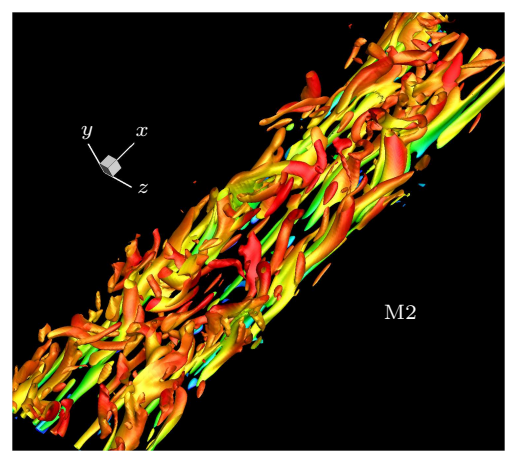

Fig. 3. Isosurfaces of the second invariant of the velocity gradient tensor $Q=0.1$ colored using average Mach number at the same instant. 
Table 3. Computational efficiency (128CPU).

\begin{tabular}{ccc}
\hline Cases & Time step & Time in per step $(\mathrm{s})$ \\
\hline M1 & $1 \times 10^{-4}$ & 2.27 \\
M2 & $4 \times 10^{-5}$ & 3.32 \\
\hline
\end{tabular}

In the near wall region, the non-dimensional mean velocity of incompressible wall turbulence shows similarity with $y^{+}$as the abscissa. When the Mach number is not high (often less than 5), Morkovin assumptions concluded that 'the essential dynamics of these shear flows will follow the incompressible pattern'. For chemical non-equilibrium compressible wall turbulence, however, it is an important problem whether similar properties exist. Figure 4(b) shows the mean velocity and Van Direst transformed velocity distributions, in which

$$
\langle u\rangle_{\mathrm{vd}}^{+}=\int_{0}^{\langle u\rangle^{+}} \sqrt{\langle\rho\rangle /\left\langle\rho_{\mathrm{w}}\right\rangle} d\langle u\rangle^{+}
$$

The log-region can be better described by Van Direst transformed velocity than another. In high speed flow, the Kármán constant $k$ still is 0.41 ; however, the logarithmic curve coefficient $C$ (6.1 for M1, 7.1 for M2) is greater than 5.1, which is different from that of the incompressible turbulent boundary-layer.
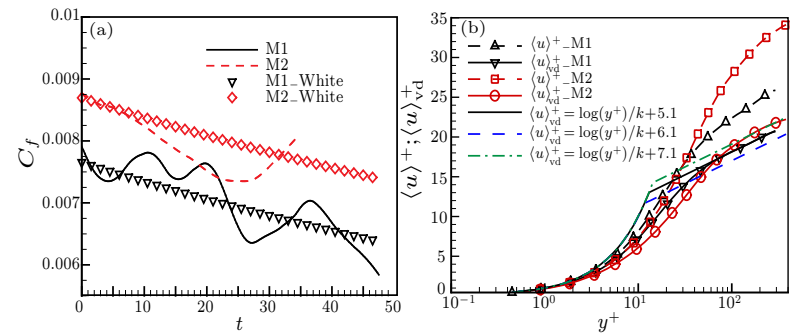

Fig. 4. (a) The relationship between skin friction coefficients and the statistical time. (b) Distribution of mean stream-wise velocity.

Table 4. Mean flow parameters for the DNS data.

\begin{tabular}{|c|c|c|c|c|c|c|c|c|c|c|c|}
\hline Cases & $\langle\rho\rangle$ & $\langle u\rangle$ & $\langle T\rangle$ & $\langle M a\rangle$ & $R e_{\tau}$ & $l^{+}$ & $\left\langle Y_{\mathrm{O}}\right\rangle$ & $\left\langle Y_{\mathrm{O}_{2}}\right\rangle$ & $\left\langle Y_{\mathrm{NO}}\right\rangle$ & $\left\langle Y_{\mathrm{N}}\right\rangle$ & $\left\langle Y_{\mathrm{N}_{2}}\right\rangle$ \\
\hline M1 & 1.11 & 0.81 & 10.41 & 1.60 & 285 & $3.5 \times 10^{-3}$ & $1.22 \times 10^{-4}$ & 0.2328 & $8.06 \times 10^{-5}$ & $1.39 \times 10^{-9}$ & 0.7670 \\
\hline M2 & 1.20 & 0.82 & 16.65 & 2.17 & 386 & $2.6 \times 10^{-3}$ & $5.87 \times 10^{-3}$ & 0.2229 & $1.13 \times 10^{-2}$ & $7.43 \times 10^{-6}$ & 0.7599 \\
\hline
\end{tabular}

Table 5. Average Damkohler number.

\begin{tabular}{cccccc}
\hline Cases & $D a_{\mathrm{O}}$ & $D a_{\mathrm{O}_{2}}$ & $D a_{\mathrm{NO}}$ & $D a_{\mathrm{N}}$ & $D a_{\mathrm{N}_{2}}$ \\
\hline M1 & $9.60 \times 10^{3}$ & 1.93 & $1.83 \times 10^{4}$ & $1.56 \times 10^{7}$ & $9.78 \times 10^{-2}$ \\
M2 & $3.34 \times 10^{5}$ & $2.54 \times 10^{4}$ & $2.99 \times 10^{5}$ & $2.87 \times 10^{7}$ & $3.00 \times 10^{3}$ \\
\hline
\end{tabular}
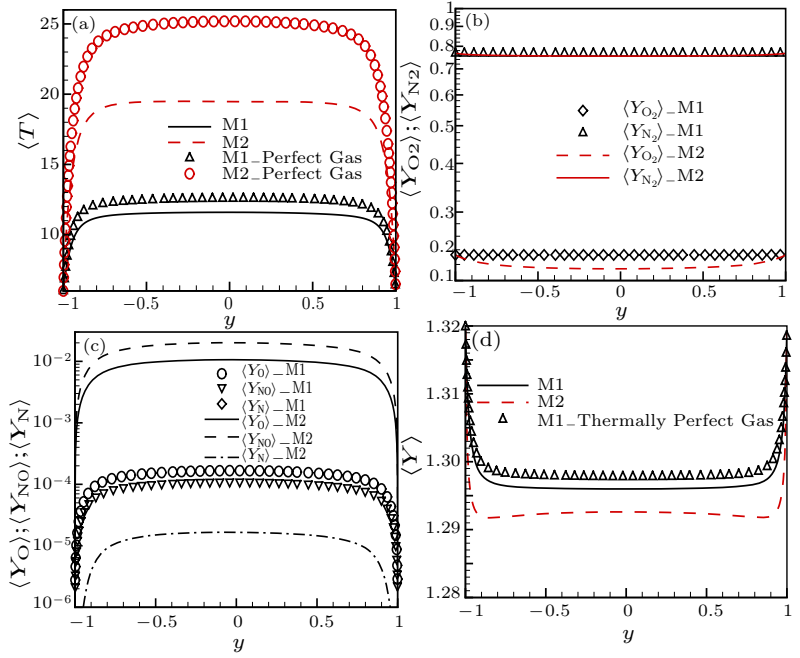

Fig. 5. (a) Distribution of mean temperature; (b) and (c) distributions of mean mass fraction; (d) distribution of specific heat ratio.

Table 4 gives the mean flow parameters of the two cases. Basic physic quantifies are expressed by the front five lists. These values are obviously different under free stream conditions, especially temperature. The mean temperature is $10.41(2255 \mathrm{~K})$ and $16.65(3607 \mathrm{~K})$, respectively; furthermore, the mean temperature profiles are given in Fig. 5(a). Figure 5 (a) also plots the result of perfect gas. It is shown that the average temperature will be reduced by the effect of chemical non-equilibrium. In contrast, the following lists are the mass fractions. In order to study the chemical reactions more easily, the mass fraction distributions in the wall-normal direction are also performed in Figs. 5(b) and 5(c). As can be seen from these results, there are not only molecular oxygen and nitrogen, but also atomic oxygen, nitric oxide and other components, which prove that the dissociation/recombination reactions happen. Because the amount of atomic nitrogen is too small, case M1 only shows four components. Meanwhile, the proportions of atomic oxygen and nitric oxide are also very small. However, in the case M2, the proportions of the atomic oxygen and nitric oxide are all significantly large. The Damkohler number $D a=\omega_{s} / \rho_{s}$ is defined as the ratio of flow characteristic time to chemical reaction characteristic time, which can be seen from Table 5 .

The maximum flow temperatures are above $3000 \mathrm{~K}$, and real-gas effects exist significantly. The specific heat ratio is one of the important parameters used to evaluate real-gas effects, which is defined as follows:

$$
\begin{aligned}
\gamma & =\frac{C_{p}}{C_{v}}, \\
C_{v} & =\sum_{s=1}^{n s} Y_{s} C_{v, s} / \sum_{s=1}^{n s} \frac{Y_{s}}{M_{s}} .
\end{aligned}
$$

Figure 5(d) shows that the specific heat ratio deviates significantly from the perfect-gas value of 1.4 throughout the wall-normal direction. From Fig. 5(d), it is obvious that the specific heat ratio of the thermally per- 
fect gas is smaller than that of the perfect gas, while even smaller than that of the chemical non-equilibrium gas.
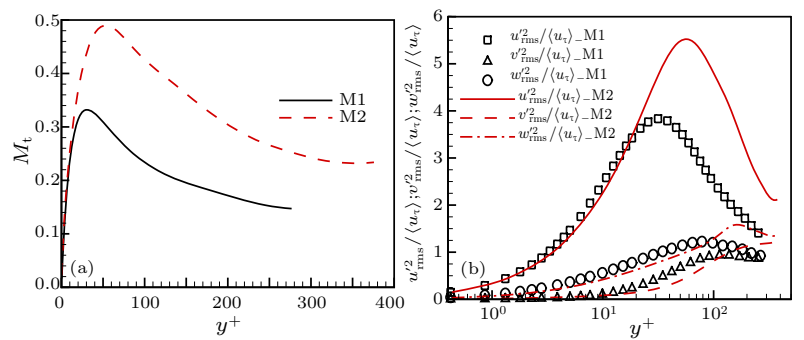

Fig. 6. (a) Distribution of turbulent Mach number. (b) Distribution of rms velocity fluctuations.

An indicator for the significance of compressibility effects is the turbulent Mach number, defined as

$$
M_{\mathrm{t}}=\frac{\sqrt{\left\langle u_{i}^{\prime} u_{i}^{\prime}\right\rangle}}{\langle c\rangle} .
$$

Figure 6(a) plots the distribution of turbulent Mach number $M_{\mathrm{t}}$ of two cases, which shows that turbulent Mach numbers are similar. The magnitude of $M_{\mathrm{t}}$ is larger than the critical value of 0.3 . Moreover, $M_{\mathrm{t}}$ (M2) is larger than the critical value in a very long area. Based on the Morkovin hypothesis, compressibility effects cannot be ignored.
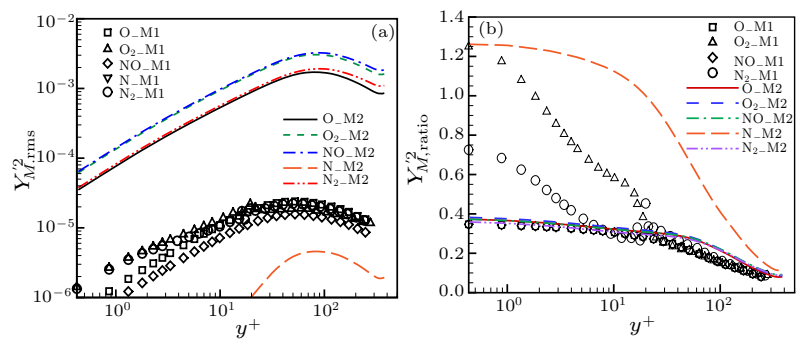

Fig. 7. (a) Distribution of rms mass fraction fluctuations.

(b) The ratio of rms and mean mass fraction distributions.

The importance of properly accounting for the mean property variations can be seen in Figs. 6(b), $7(\mathrm{a})$, and $7(\mathrm{~b})$. When normalized by conventional wall variables (defined in terms of the mean density, viscosity and shear stress at the wall), rms velocity profiles have little to do with inflow Mach number near the wall. However, they increase with the inflow Mach number away from the wall. Figure 6(b) plots the rms mass fraction fluctuations of five different components, which increase with the inflow Mach number. The rms (M1) of $\mathrm{N}$ component does not display because it is too small. In order to analyze the fluctuation effects, a ratio will be defined as

$$
Y_{M, \text { ratio }}=\frac{Y_{M, \mathrm{rms}}^{\prime 2}}{\left|\left\langle Y_{M}\right\rangle-Y_{M, 0}\right|},
$$

where $Y_{M, 0}$ is the mass fraction conditions in the wall. The ratios have been plotted at Fig. $7(\mathrm{~b})$. It can be seen that the ratios are similar, which decrease with the increase of $y^{+}$. The values are all about 10-35\% except molecular oxygen (M1), nitrogen (M1, M2), and molecular nitrogen (M2). The ratios, does not in the range of $10-35 \%$, can mainly cause by their change are small.

In summary, based on the assumption of local thermal equilibrium and chemical non-equilibrium, we develop a high-order code and then verify the code by a numerical test of non-equilibrium cone flow. By using the code, we perform direct numerical simulations of hypersonic and high-temperature turbulent channel flows, which inflow Mach number at 6 and 10. In fully developed turbulent flow, the skin friction coefficient is closely analogous to the White correlation. The log-region can be well described by Van Direst transformed velocity. The temperature will rise in the near wall region quickly. The values of specific heat ratios are between 1.4 (perfect-gas) and 1.286 (vibrational excited completely), which proves that the dissociation/recombination reactions begin to emerge and become more and more important. The turbulent Mach number reveals that compressibility effects need to be considered. The rms velocity profiles have little to do with inflow Mach number near the wall. However, they increase with inflow Mach number away from the wall. The rms mass fraction fluctuations increase with inflow Mach number, and account for a significant proportion of mass fraction.

\section{References}

[1] Reed H 2009 RTO-EN-AVT-151 13. 1-13.15 (van Karman Institut, Rhode -Saint-Genese, Belgium)

[2] Anderson J D 2000 AIAA (New York)

[3] Martin M P 2003 AIAA Paper 03-4055

[4] Martin M P 2007 J. Fluid Mech. 570347

[5] Martin M P and Candler G V 1998 Phys. Fluids 101715

[6] Martin M P and Candler G V 1999 Phys. Fluids 112765

[7] Duan L and Martin M P 2009 AIAA Paper 09-4040

[8] Duan L and Martin M P 2009 AIAA J. 47244

[9] Duan L and Martin M P 2011 AIAA J. 49172

[10] Duan L and Martin M P 2011 J. Fluid Mech. 68425

[11] Marxen O, Iaccarino G and Shaqfeh E S G 2007 Annual Research Briefs (Center for Turbulence Research, Stanford University)

[12] Marxen O, Magin T, Iaccarino G and Shaqfeh E S G 2010 AIAA Paper 10-0707

[13] Chen X P, Li X L and Fan J 2011 Sin. Phys. Mech. Astron. 41969 (in Chinese)

[14] Gupta R N, Yos J M, Thomspson R A and Lee K P 1990 $N A S A-1232$

[15] Gnoffo P A, Gupta R N and Shinn J D 1989 NASA-TP2867

[16] Barbante P F and Magin T E 2004 RTO-EN-AVT-116

[17] Park C 1985 AIAA Paper 85-0247

[18] Park C 2000 AIAA Paper 00-0210

[19] Park C 2001 J. Therm. Heat Transfer. 1576

[20] Jiang G S and Shu C W 1996 J. Comput. Phys. 126202

[21] Kyu H and Chonggam K 1998 AIAA Paper 98-2442

[22] Zhang Z S, Cui G X and Xu C X 2005 (Beijing: Tsinghua University Press) (in Chinese)

[23] White F M 1974 Viscous Fluid Flow (New York: McGrawHill) 